nor for whom, with the present appropriation, can a place be provided.

2. Earnest and intelligent co-operation with the local school board to provide an outdoor school or fresh-air room for pre-tuberculous children; if such a school or room is established, the attention of the city legislature and board of health should be called to the good which it does.

3. It should be insisted upon that the terms of the 1908 act, that "tuberculosis and its prevention shall be taught in our public schools," stc., be complied with. This can best be done by means of tuberculosis exhibits prepared along the lines of those now loaned by the state, placed in all the public schools, and demonstrated whereever possible by doctors, nurses or social workers.

I know of no more fundamental or important work for any anti-tuberculosis society to take up than this.

\section{THE THERAPEUTICS OF' LIGHT.*}

BY HERBERT MC INTOBF, A.M., M.D., BOs'ON.

THE pioneer work of Finsen has served to direct attention in recent years to the utility of light therapeutics. Though his labors were chiefly confined to the treatment of lupus, in the control of which he achieved brilliant success, later students have found that light is applicable to the treatment and cure of a wide range of troubles. The purpose of this paper is to indicate, first, why, upon theoretic grounds, it is fair to conclude that light should exert an important effect upon the cure of disease, and, secondly, to indicate in a general way some of the uses to which it may be put.

It is scarcely necessary to say by way of introduction that light is the condition of terrestrial life. Whatever the life principle is, and it is still a profound mystery, it cannot manifest itself except under conditions made possible by light, and in the preparation of the earth as the habitation of man the precedent evolution of the vegetable kingdom by light was essential.

What a mighty force sunlight is, is made manifest when we reflect that by means of evaporation millions of tons of water are daily lifted into the atmosphere to descend as rain, and fill the water courses which in turn will ultimately be called upon, as the coal in our mines - the product of the sun's mighty activity in the carbonaceous period - is consumed, to supply power for industrial and domestic uses.

In like manner the same silent energy in the springtime lifts the sap from the roots into the trunks and branches of trees and into the stems of countless forms of vegetable life; in the aggregate an uncomputed weight of life-bearing fluid.

The influence of sunlight upon protoplasm is illustrated by many chemical phenomena. Thus bilirubin is oxidized into biliverdin by sunlight even when air is excluded and $\mathrm{cl}_{2}$ unites with $\mathrm{C}_{2} \mathrm{cl}_{4}$ in sunlight forming $\mathrm{C}_{2} \mathrm{cl}_{6}$. Chlorine unites with hydrogen in sunlight with explosive violence to form hydrochloric acid.

* A paper read before the Brookline Medical Club, Nov. 9, 1910.
The fermentative effects of light are very notable. Thus Niepce de Saint Victor and Corvisant found that a $0.1 \%$ solution of starch exposed during six or eight hours to the summer sun becomes transformed into sugar, no change occurring in the dark. In like manner glycogen is transformed into sugar more rapidly in the light than in the dark.

Passing now into the vegetable world, we find that under the influence of sunlight there is formed in the phanerogams or green-leaf plants a product which is called chlorophyll, by means of which the plant is enabled to break up the molecule of carbon dioxide and fix carbon in the form of starch into its own structure, thereby supplying an indispensable food for the sustenance of animals as well as material for the construction of dwellings.

This too brief review of the physical and physiological effects of light in nature prepares us to consider what effect it may have upon human life.

\section{ABSORPTION OF LIGH'T BY BLOOD.}

Freund squeezed a drop of blood from the tip of his finger upon a quartz film, surrounded by a rim of paper $0.17 \mathrm{~mm}$. wide, and covered it with another quartz film. The layer of blood completely filled the space of $0.17 \mathrm{~mm}$. and showed uniformly red. The spectrum was photographed through a glass spectroscope by means of sunlight. The exposure lasted five minutes and produced no effect upon the photographic plate from lines F $\frac{1}{2} \mathrm{G}$ in the direction of the ultraviolet.

A second experiment was made upon the web of a live frog's foot in which the blood stream was flowing. The foot was placed in front of the slit of the spectroscope and illuminated by sunlight. The exposure lasted five minutes. Under these circumstances the line $H(\lambda=3,964 \ddot{A})$ was absorbed. This is very near the edge of the ultraviolet. From this experiment it is clear that blood absorbs light. It is for this reason that Finsen found it necessary in his treatment of lupus to employ compression upon the area treated in order to squeeze out the layer of blood which acted as a screen to the chemical rays.

Finsen's historic experiment with aristo paper placed behind the lobe of his wife's ear and illuminated for five minutes by the concentrated light of an arc lamp established the same fact. Without compression there was no effect upon the paper, but when the ear was compressed between two glass plates until it appeared bloodless, the aristo paper was blackened after an exposure of twenty seconds. From these experiments wo may safely infer that blood absorbs light.

Quincke ${ }^{1}$ found that hemoglobin gives off its oxygen more quickly in the light than in the dark. Therefore, light increases the oxidation of the tissues.

This action is somewhat analogous to the influence of light upon the molecule of carbon dioxide in the presence of chlorophyll. Here the atom of carbon is dissociated from the bivalent

1 Pfuger's Archives, 1894, vol. Ivii, p. 134. 
oxygen which is released, while the carbon in the form of cellulose is fixed in the cellular structure.

Fluorescence is the property which some substances have of absorbing higher frequencies and converting them into lower frequencies. Among these substances are blood and serum. These are, therefore, radiant energy transformers.

What is the significance of this fact that blood and serum like chlorophyll are fluorescent? It has been frequently affirmed, and correctly, that the ultraviolet rays have little penetration, and yet it is generally believed that thesc rays possess the highest virtue in phototherapeutics. It is certain that Finsen attached the greatest value to them in his therapeutic studies. If, now, they have but slight power to penetrate the skin, their value must be limited to very superficial conditions. Here we are met, however, by the fact discovered by Dr. Henry Bence Jones, in 1866, that blood and serum are fluorescent media. By this is meant that they have the property of absorbing light at one rate of vibration, as, for example, ultraviolet, and transforming it into a lower rate. They are, therefore, transformers of radiant energy, and we are justified in assuming that the absorption of the rays of higher refrangibility by the epidermis is not synonymous with their extinction, but rather with their transformation into another rate of light energy.

Having shown, then, that the ultraviolet frequencies which have little power of penetration are absorbed by the blood stream and have the ability to produce fluorescence and thus profoundly affect the chemistry of the tissues, let us turn our attention to the visible chemical frequencies. These include the upper end of the visible spectrum marked by the blue, indigo and violet. Now, it has been frequently proved that these chemical frequencies of the visible spectrum have marked power of penetration.

Thus Gadneff introduced under the skin of cats and dogs small tubes containing silver chloride. After exposure to sunlight the chloride of silver was found to be blackened, while no change occurred with captive animals kept in the dark.

Darbois ${ }^{2}$ put a piece of photographic paper between two watch glasses. He then placed the watch glasses in the mouth against the buccal membrane. The light from a Finsen concentrator was then thrown upon the cheek. The photographic paper was blackened after one minute.

Gebhard ${ }^{3}$ imbedded his hand in plaster of Paris, leaving only the back exposed, having placed a photographic plate in the palm. The hand was exposed for twenty minutes to the light of an electric arc lamp. The plate was afterwards developed and showed blackening with outlines of hand and fingers.

Solucha ${ }^{4}$ placed strips of silver bromide gelatine under the skin of dogs and sewed up the wounds. The light from a ten to twenty-ampere lamp at a pressure of from fifty tocixty-five volts was thrown upon the parts with the result that decomposition of the silver bromide occurred in one half a minute.

Traitement du Lupus Vulgaire. Thèse de Paris, 1910, p. 80. Die Heilkraft des Liohtes, Leipsig, 1808, p. 131.

- Quoted by Freund.
When the tubes were placed behind the ear or in the mouth against the check, decomposition soon occurred. When, however, the tubes were placed decp in the gluteal muscles, the light had no effect. When placed behind the forearm or in the closed hand there was no decomposition after fifteen minutes.

When, however, the current was increased to twenty amperes at 110 volts, the sensitized gelatine film was ehemically altered when placed on the back of the neck or on the side of the body, the rays being thrown perpendicularly upon the film through the tissues.

I placed an x-ray film back of a dental skiagraph and put them between two watch glasses in a dark room. They were then introduced into the mouth between the teeth and the cheek. I then had the light of an arc 2,000 candle-power lamp thrown upon my check for about ten minutes and secured a very good picture of the dental skiagraph upon the sensitized film. This was obtained from the rays passing through the check. We have thus shown that visible chemical frequencies penetrate the body, and that the ultraviolet rays through their ability to excite fluorescence in the serum and blood stream are converted into chemical rays of lower frequencies.

That the luminous frequencies are capable of penetration is evident from the simple diagnostic procedures employed in the different specialties. Thus a 4 candle-power lamp placed in the mouth is capable of illuminating the maxillary antra; placed in the superior anterior angle of the orbit is capable of illuminating the frontal sinus; when introduced into the stomach by means of the gastrodiaphane illuminates the epigastric and surrounding regions; and when carried into the vagina irradiates an area extending up to the umbilicus.

From this brief survey of the field it is elear that all the frequencies penetrate the tissues, not only the red, yellow and orange, but the highly useful chemical frequencies of both the visible and invisible regions.

Which of these radiations are the most useful in therapcutics? Here again we recognize a general agreement as to the value of the heat waves, so that no need exists to emphasize the importance and value of the rays of greater lengths. Both theoretically, however, and practically, their utility is much less than that of the high rates of vibration. It is only as the wave lengths begin to approximate the diameter of the molecule that the most profound effects are discernible. Thus, while in plant life it is the yellow rays that are chiefly concerned in the function of assimilation, breaking up the carbon dioxide molecule, depositing carbon in the cellular structure and releasing oxygen, it is to the chemical rays that all excitomotor phenomena are due. The rays of greater wave length pass through the tissues practically without change except to impart a portion of their heat to the structures upon which they impinge. In like manner a wave upon the surface of the water is not destroyed 
by an object of smaller size, but will fold itself around it and pass on unbroken, but if it be near the size of the obstructing object it communicates its vibration to the opposing object and is destroyed. In like manner the shorter vibration of the chemical portions of the spectrum surrender their energy in the tissues upon which they strike and thus produce their beneficial effects.

The absorption of oxygen by the blood under the influence of the chemical rays and the fluorescence of blood and serum under the influence of the more refrangible portion of the spectrum go far toward establishing the proposition that it is to the upper end of the spectrum that we must attribute the peculiar value of light treatment. I desire to emphasize this point because it is frequently affirmed by those who have given little consideration to the therapeutic uses of light that the value of light treatment resides in the impartation of heat to the tissues. This is measureably true. With the exception of thermopenetration, which has lately been introduced to the profession and which raises the temperature of the tissues in the path of the high-frequency current from eight to ten degrees, there is no method comparable to light for the production of heat in the tissues. The incandescent bath produces sweating in half the time required by a Turkish bath. This is in spite of the fact that the air in an incandescent bath is but little above the temperature of the body. In a Turkish bath the body is enveloped by a blanket of hot air and the temperature of the body is raised by actual contact with the source of heat. In an incandescent bath the radiant heat and light rays from incandescent bulbs traverse the air without impartation of energy, impinge upon the body, penetrate the tissues and produce physiological changes. The chemical portions of the spectrum undoubtedly produce metabolic effects, though it is usual to regard the incandescent bath as primarily a heat bath. It is in any event a much more agreeable form of heat bath than the Turkish bath, and patients quite invariably prefer the incandescent bath to the ordinary hot air bath.

While, however, it is to chemical rays that the peculiar value of light treatment must be ascribed, for general purposes the whole spectrum of white light is to be preferred. There can be no objection to using, and in most cases there is great value in including, the luminous and heat rays in the treatment of disease. Special indication, however, may call now for the exclusion of the heat rays and now for the exclusion of the ultraviolet rays. For general purposes, however, the full spectrum of white light, such preferably as is furnished by an 2,000 candle-power arc lamp, should be employed, and when only one lamp is used, there can be little doubt that this furnishes the best result. It is not denied, however, that the incandescent lamp of high candle power is a very useful agency for general therapeutic purposes.

Having directed attention to some considerations which should lead us to infer that light should a priori exert a favorable influence upon disturbed bodily conditions, let us sum up briefly some of the physiological effects of light which have been experimentally proved.

It is a matter of common observation that the skin may become irritated under the influence of the sun's rays and sustain burns under continued exposure which may produce great discomfort. Thus solar erythema and snow blindness are illustrations of the irritant effects of the ultraviolet rays of the sun.

Similarly, the tanning of the skin in summer time testifies to the influence which the sun's rays exert upon the pigment-producing cells of the rete mucosum.

Sweating occurs much more rapidly in an incandescent bath cabinet than in a Turkish bath, yet the temperature in the latter is much higher. Light, therefore, has a marked influence upon the sweat glands.

The effect of the impingement of light rays upon the body is to increase superficial hyperemia, and as a determination of blood to the surface cannot take place without its withdrawal from the interior, it follows that when large areas of the body are exposed to light energy there must be a depletion of the viscera.

light energy is destructive of bacterial life outside the body. Since, however, this destructive action is due chiefly to the ultraviolet rays, and these are not highly penetrative, we are not permitted to affirm a directly bactericidal action in living tissue. I have already pointed out, however, that blood absorbs light, that the tissues fluoresec under the influence of light, and that the hemoglobin gives up its oxygen to the tissues more readily under light exposure. Herein may be perceived the damaging effect of light upon bacteria in the tissues. For as they are anaerobic, the production of oxygen is unfavorable to their growth and the increased activity of tissue combustion produced by light renders the nutrient media in which they live less favorable to their propagation.

The increased absorption of oxygen by the blood under the influence of light leads to increased activity in tissue destruction, and, therefore, augments the elimination of $\mathrm{CO}_{2}$. In confirmation of this proposition Molschott, ${ }^{5}$ Selmi and Piacentini ${ }^{6}$ have shown that dogs, hens, pigeons and frogs eliminate less carbon dioxide in the dark than in the light.

In like manner S. Goodnow has shown that human beings and animals secrete more urine, urea and chlorides when continually exposed to daylight than when remaining in the dark. We may thus fairly conclude that light energy increases urinary elimination.

When the nude body is exposed to light energy there is a rise in temperature and an acceleration of both pulse and respiration. These observations of Goodnow have been repeatedly confirmed by the writer. Thus in a recent case treated in the incandescent bath for obesity, the temperature quickly rose from normal to $101^{\circ}$, and the pulse from 80 to occasionally 140 . We may conse-

"Wien. Med. Wochenschr., 1885, no. 43.

- Rund. Conti. del IRealo Instituto, 1870, vol. iii, Scr. ii, p. 51. 
questly affirm that light tends to raise the normal temperature and accelerate the pulse-rate. It should be remarked, however, that these results are obtained from the whole spectrum, colored rays tending to lower the pulse, particularly the violet.

Sudden exposure to bright light may produce violent sneezing. This is a reflex from the sensory fibers of the trigeminus distributed to the Schneiderian membrane. Hence we may conclude that light energy may produce motor excitation.

Light markedly increases the number of red blood cells. This has been repeatedly demonstrated after an incandescent bath. It also increases the amount of hemoglobin. Under the influence of light oxyhemoglobin more readily surrenders its oxygen to the tissues, thereby stimulating combustion of the tissues. We have already shown that the solid constituents of the urine are increased, that sweating is promoted and that the elimination of $\mathrm{CO}_{2}$ is augmented.

Cretinism occurs in the dark valleys where the rays of the sun never penetrate, and amenorrhea afflicts the Eskimo women during the long Arctic night. It is very evident, then, that sunlight promotes metabolism.

It would be profitable to spend some time, if it were at my disposal, upon the interesting subject of blue light. Many of you will recall the interest excited among the laity, in the 70's of the last century, by the publication of two books upon the use of colored lights. The first, published in 1876 by Gen. A. J. Pleasonton, of Philadelphia, a veteran of the Civil War, is entitled "The Influence of the Blue Ray of the Sunlight." The other is entitled "Blue and Red Light, or Light and its Rays as Medicine." It was written by a physician, Dr. S. Pancoast, of Philadelphia, and published the following year. These books have for us to-day simply an antiquarian interest. The first is highly fantastic, and neither has much scientific value. They excited, however, a great deal of interest, and all over the country houses were supplied with colored lights; generally blue or violet, sometimes alternate red and blue. On Beacon Street opposite the Common you may still see several stately dwellings ornamented by colored lights which are a memorial of the interest excited by these early writers. These books are now out of print, but may be found in the Boston Public Library.

The fact remains, however, that there is a therapeutic value attached to the blue and the red ray. 'Thus it is undeniable, that the blue ray exerts a constringing effect upon the blood vessels and a soothing influence upon the peripheral nerves, and there is now a general agreement that the blue light is analgesic and in some instances anesthetic, and much clinical proof can be furnished to sustain this proposition. For the relief of superficial neuralgias, as, for example, trigeminal neuralgia, the results are sometimes very astonishing. Myalgia and arthritis similarly often yield promptly to the blue ray. Von Jaksch ${ }^{7}$ dwells upon the soothing effect of blue

\footnotetext{
' xx. Congress fur Innero Medizin, 1902.
}

light and uses blue chimneys upon his lamps in his sick wards. The healing of wounds is promoted by the blue light, and Pansini has shown by an elaborate study fortified by ergograms that blue light increases the power to do muscular work. The efficacy of red light in smallpox depends upon the exclusion of the blue ray rather than upon any virtue residing in the red ray. The chemical ray promotes suppuration. If, therefore, the blue ray is excluded, pitting is prevented and the comfort of the patient promoted by the admission of red light. The use of the red ray in smallpox is associated with the name of Finsen, who was an enthusiastic advocate of this method of managing variola. He was not, however, the first to employ red light in this disease. John, of Gaddesden, ${ }^{8}$ who died in 1361 , was the author of the first medical treatise in the English language. The son of King Edward I, who was afflicted with smallpox, was under his care. He was placed in a bed with scarlet hangings and red blankets and counterpane. His throat was gargled with mulberry wine and his mouth washed with the juice of red pomegranates. The royal patient recovered without the usual disfigurements attending this dread disease. In Queen Elizabeth's time the value of red curtains and red glass was strongly affirmed by certain physicians, who, however, were regarded by the orthodox physicians of the day as charlatans.

Finsen, while reading in the library at Copenhagen, ran across an account of some sailors, suffering from smallpox, who were confined in a darkened room at New Orleans and recovered without pitting, and was thus led to recommend the exclusion of the chemical rays in the treatment of this disease. Finsen ${ }^{\circ}$ condemns unsparingly the treatment of smallpox cases without the exclusion of the chemical frequencies of light.

Apart from these obvious uses of colored lights, there is undoubtedly a psychical aspect to the problem. Blue is a so-called "quiet color." It is associated with the quiet of the evening, the calm of the twilight and is the symbol of peace. Red, on the other hand, is a so-called "loud color" and is associated with the activity and bustle of the day and is the symbol of conflict and passion. The bull is maddened by it; unchaste women select it as the emblem of their unhallowed traffic, and the ancient temples of Venus were richly adorned with it.

More or less study of the effects of colored lights upon insane patients has been made, somewhat confirmatory of the conclusions already noted. Maniacal patients have, in some instances, been quieted by blue lights, while those afflicted with melancholia have been to some extent relieved by red. At the present stage of experimentation it would not be safe to affirm either uniform or positive results. In some instances they have been negative, as in the experiments of Von Paquet of Moscow.

The value of sunlight in surgery is well illustrated in a paper read at the Second International

8 Phila. Med. Jour., Dec. 7, 1901

- Brit. Med. Jour., Juno B, 1903. 
('ongress for Physiotherapy at Rome, Oct. 13-16, 1908, by Dr. Oskar Bernhard Moritz. ${ }^{10}$ The remarkable results which this'surgeon has attained in the healing of wounds, ulcers and skin affections he attributes $(a)$ to the increased proportion of ultraviolet rays contained in the sunlight of elevated regions. Thus Oberengadin in the Alps, where the results reported were secured, is 1,800 meters above the sea. Here the weakening of the sun's rays through filtration through the atmosphere is enormously decreased and the ultraviolet rays possess greatly increased power. It is to this circumstance that Dr. Moritz attributes the freedom of the mountaineers of the high Alps from lupus and skin cancer, affirming that a chronic solare eczema renders the skin resistant to the invasion of bacteria. (b) To the remarkable dryness of the atmosphere in these elevated regions. Here again, however, we have an explanation in the increased ultraviolet content of the sun's rays, since even small quantities of water vapor act as a screen to the passage of the ultraviolet ray; and $(c)$ to the absence of bacteria from these elevated regions. A little reflection here will again make clear that the absence of bacteria from mountain air is due to the increased proportion of ultraviolet rays in mountain sunlight.

Dr. Moritz cites the remarkable effect of sunlight upon a wound made in extirpating the spleen, which had broken open on the eighth day after operation, holding only by the peritoneal edges. In spite of curetting and cauterizing it had made no progress in healing. After an hour and a quarter's exposure to sunlight there was a distinct improvement, the granulations were markedly better and stronger and the great gaping wound under this treatment healed quickly. In grafting he simply lays the grafts in the sun upon the dry granulating surface. Within a quarter of an hour it is usually impossible to detach the grafts from the underlying tissue without exerting considerable force.

In like manner drainage becomes unnecessary for the most part in deep wounds, since insolation both dries out the deep pockets and incidentally destroys bacteria. Ulcers and cavities become through insolation in a short time pure and dry. 'They are quickly covered with a shiny fine parchment-like skin which usually lasts for from twenty to thirty hours, when the wounds are again exposed to sunlight. Any one who desires may readily verify these statements. A wound exposed to sunlight or the electric arc is quickly covered with a parchment-like membrane and the edges are crumpled and drawn together. Frequently no dressing is required.

With equally favorable results Dr. Moritz employs the insolation method in cases of tuberculosis of the skin, glands, joints, bones and larynx.

It is important to observe that in the method of Dr. Moritz, the rays of the sun are not concentrated, neither is there any exclusion of the

10 Jahrbuch aber Leistunuen und Fortsehritte auf dem Gebiete der physikalinchen Medizin, 1008, p. 77. heat rays, for which he claims under proper conditions both chemical and bactericidal power. In the winter the direct rays of the sun are reinforced by reflection from snow and ice. He reports cases of scrofuloderma which had resisted all other therapy for three years but yielded to sunlight treatment after three months, and two severe cases of lupus, one of which had involved the soft palate and the vocal cords. In cases of bone and joint tuberculosis he combines insolation and immobilization, placing the disabled part in a plaster of Paris jacket and exposing it to the sun's rays through openings cut in the dressing. Among the other surgical conditions for which sunlight treatment is valuable, he mentions crushing wounds, ulcera cruris, perforating ulcer of the foot, wounds caused by infection, as abscesses, paronychia, furuncle, carbuncle, soft ulcers, buboes, and syphilitic ulcers associated with specific treatment.

It is possible that the very remarkable results obtained at high elevations could not be secured at lower elevations, and that there is a peculiar virtue in sunlight at these high altitudes. It is nevertheless true that sunlight treatment can be employed with advantage in many cases which are carefully excluded from the light by dark dressings, and particularly in those cases which have shown themselves rebellious to ordinary treatment.

It remains for me to state briefly the apparatus required for light treatment. The most generally useful apparatus for office use is in my opinion the arc lamp of 2,000 candle power. This furnishes the whole spectrum, including the ultraviolet rays, upon the importance of which I have sought to lay emphasis in the preceding pages. It furnishes a tremendous volume of rich chemical rays both visible and invisible, as well as abundant heat rays. Screens may be used to furnish blue or red light.

A substitute for the arc lamp is the high power incandescent lamp. Here, however, the ultraviolet rays are entirely cut off by the inclosing bulbs, and the treatment is necessarily to a large extent a radiant heat treatment. This is, of course, of great value, but manifestly inferior to a treatment which utilizes the whole spectrum as does the arc light.

Next there is the incandescent bath cabinet, a most useful apparatus, in which the nude body of the patient is exposed to the radiance of fifty or more sixteen-candle-power incandescent bulbs. With this apparatus it is necessary to combine certain hydriatic procedures in order to produce a suitable reaction and prepare the patient for exposure to ordinary temperatures.

The are bath cabinet is also a highly useful apparatus. This is a cabinet six or more feet in length and of suitable width and height. It contains a cot upon which the patient lies nude and exposed to the rays from two powerful arc lamps. It is an artificial sun bath.

For dermatological uses particularly, there are lamps after the pattern of the Lortet et Genoud lamp designed to take the place of Finsen's enormous concentrators. These have quartz 
windows to permit the escape of the ultraviolet rays so important in most therapeutic treatments. Then there are small lamps with iron or other metal electrodes and quarty windows which are almost exclusively ultraviolet lamps.

Finally, there is the Kromayer mercury vapor lamp in which a current of electricity is passed through volatilized mercury, producing a peculiar greenish-blue light. This is provided with quartz windows for the egress of the ultraviolet rays. It has been used chiefly abroad for therapeutic purposes and has recently been adapted to the sterilization of potable water. It is attached to the water faucet and automatically and absolutely destroys all bacteria, rendering water thus irradiated safe for domestic purposes.

Summary. In this brief sketch of the therapeutic uses of light, I have endeavored to show

(a) That light penetrates the tissues.

(b) That in so doing the chemical rays produce fluorescence of the blood and serum which is reduced or absent in pathological states, as, for example, in malaria.

(c) That the action of light upon the blood stream reduces oxyhemoglobin and thus stimulates the chemistry of the tissues, that is to say, improves metabolism.

(d) That the most useful portion of the spectrum is not the heat or luminous portion, but the chemical portion.

(e) That the physiological effects of light flow casily from the marked penetration of the light rays, particularly the chemical portion of the spectrum.

(f) That the blue light has a peculiar field in the control which it has over pain and hyperemia.

(g) That red light by its exclusion of the chemical rays plays an important rôle in the treatment of smallpox.

Finally, I have given some illustrations of the efficient action of light, of artificial sunlight, and concluded with a brief description of the apparatus employed in light therapy.

My aim has been principally to show that the use of light for therapeutic purposes is rational and that it is a most important addition to the physician's equipment for the management of disease.

\section{FiXTRAPERITONLAL CAESAREAN SECTION.}

BY JAMES LINCOLN HUNTINOTON, M.D., BOBTON.

To realize why this operation was revived and why it was received with such enthusiasm in Germany, one must first bear in mind the condition of German obstetrics. Normal labor is there conducted almost entirely by midwives, who are required by law to call in the obstetrician when complications arise. This results in what we might call ultra-conservative obstetrics cases come late to operation. The midwives, in spite of their thorough training in the schools under the supervision of the government, and their rigid examinations, are far from being keen diagnosticians or careful practitioners. The number of fatal cases of puerperal fever in Germany annually is still over five thousand. The first indication for operative interference taught the German student in obstetrics is fever during labor (a condition very rarely met with here in America). This is the commonest complication for which the obstetrician answers the cry for help from the German midwife; he comes and finds all too often on examination a contracted pelvis, os fully dilated, membranes ruptured and head above the pelvis brim. What is to be done? The answer was, and still is, in most German Kliniks, craniotomy. Yes, craniotomy on the living child has been far more common in Germany than here in America, and it is because of the frequency of infection during labor for which the midwife is the most constant contributing factor.

But if any one has done this operation, or even seen it executed, he will understand with what feverish ardor the obstetricians in Germany sought for a solution which would save them from performing this hideous operation. The operation of widening the pelvis was looked upon with favor for these cases until the fatalities following cven the smallest wound in the vagina - and sometimes apparently without this break in technic - forced the obstetricians to regard infection as the strictest indication against this operation. Then the indefatigable researches into the realm of surgical possibilities revived this forgotten operation of entering the gravid uterus through a wound shut off from the peritoneal cavity.

Before deciding whether or not this operation has solved the problem they were facing, let us review the history of this surgical procedure.

In 1807 Jörg, viewing the high mortality of Casareans in the days before antiseptics, conceived the idea of a modified Casarean section by opening the vagina and cervix through a low lateral abdominal incision. Acting on his suggestion Ritgen modified this by not opening the vagina, but pushing the bladder to one side and opening the uterus extraperitoneally. In 1821 we have the account of his first attempt at such an operation an attempt merely, because of uncontrollable bleeding which caused him to change the operation to the classical Casarean to save the child's life. The patient died two days later from hemorrhage.

In 1822 Physick, of Philadelphia, in a letter to Dr. Horner, proposed a similar operation, only that he used a transverse incision above the symphysis pubis instead of the median longitudinal incision which Ritgen employed. Again in France in 1823 we have an account of a similar attempt at an extraperitoneal Casarean changed during the operation to the classical because of difficulties.

In 1870 Dr. T. G. Thomas, of the College of Physicians and Surgeons in New York, ignorant of previous work along this line, conceived the idea of an extraperitoneal operation and performed such an operation on the cadaver of a young woman who died at term. He found the operation so simple that he resolved to employ it upon the next favorable opportunity. Shortly after this 